\title{
Unprotected synthesis
}

Chemists at The Scripps Research Institute demonstrate that it is possible to synthesize complex natural products without the use of protecting groups.

One of the fundamental tenets of modern organic chemistry is the use of protecting groups in the total synthesis of complex molecules such as natural products. Phil Baran, a chemist at Scripps, likens their use to donning a molecular 'winter coat', allowing one functional group to be addressed individually while protecting other functionalities from unwanted reactions. "A general problem [in total synthesis] is that we don't know how to control chemoselectivity the way nature does," explains Baran.

But what would happen if one tried to design a synthesis of a complex natural product without protecting groups? That is just what Baran and his colleagues showed in a recent paper (Baran et al., 2007). By applying eight general guidelines for retrosyn- thetic analysis, they designed novel syntheses for four molecules from different classes of natural products. By avoiding the use of protecting groups, they were forced to come up with new chemoselective chemical transformations. "If your purpose is just to make a molecule and you don't care how it's made, go ahead and do it the old way, but [try this strategy] if your desire is to understand something new about chemistry and to challenge yourself to invent something," says Baran.

Additionally, with moves that may prove to be of particular interest to the pharmaceutical industry, Baran and colleagues were able to reduce the number of synthetic steps (in some cases, from more than 20 to less than 10 ), and obtain a higher overall yield of the desired final product. He is quick to point out, however, "that this [strategy], of course, is not something that you can generally apply to everything on the planet, and I'm certain that certain classes of natural products will always need protecting groups." Nonetheless, Baran hopes that that their recommended guidelines will inspire synthetic chemists to invent new methods for chemoselective transformations and drive the science of organic chemistry forward.

Baran quickly followed up with a second paper (O’Malley et al., 2007), demonstrating that the protecting group-free strategy worked for a completely different natural product family and providing further proof that it may indeed find widespread applicability in the field of total synthesis. Says Baran: "Expect more examples in the future!"

\section{Allison Doerr}

\section{RESEARCH PAPERS}

Baran, P.S. et al. Total synthesis of marine natural products without using protecting groups. Nature 446, 404-408 (2007).

O'Malley, D.P. et al. Total synthesis of dimeric pyrrole-imidazole alkaloids: sceptrin, ageliferin, nagelamide $E$, oxysceptrin, nakamuric acid, and the axinellamine carbon skeleton. J. Am. Chem. Soc.; published online 22 March 2007. 